\title{
Collective phenomena in high-energy nuclear collisions
}

\author{
Jiangyong Jia \\ Department of Chemistry, Stony Brook University, Stony Brook, NY 11794, USA, \\ Physics Department, Brookhaven National Laboratory, Upton, NY 11796, USA
}

\begin{abstract}
I review experimental studies of collective phenomena in $p+\mathrm{A}$ and $\mathrm{A}+\mathrm{A}$ collisions presented in the Quark Matter 2014 conference. Keywords: Heavy-ion collisions, Fluctuations, Correlations, Collective flow, Ridge
\end{abstract}

\section{Introduction}

The goal of high-energy nuclear experiments is to understand the properties of the QCD matter created in the heavy-ion collisions. Most progress to this end has been obtained from study of collective flow phenomena, which are sensitive to the dynamics of the system at various stages of the space-time evolution. Extensive measurements of various flow observables in A+A collisions at RHIC and the LHC, in combination with successful modeling by relativistic viscous hydrodynamics, have placed important constraints on the transport properties and initial conditions of the produced matter $[1,2]$.

Recently, qualitatively similar collective behaviour has also been observed in high-multiplicity $p+$ A collisions [3]. Current debate is focused on the effective mechanism behind the apparent collectivity in these small systems: Is it initial state effect, final state effect, or both?

This contribution reviews the new results from $p+\mathrm{A}$ and $\mathrm{A}+\mathrm{A}$ collisions from RHIC and the LHC, obtained with various flow observables. I first summarize experimental progresses in studying the collectivity in small collision systems. I then make a few remarks on flow results in A+A collisions that I personally found interesting. This is followed by a focused discussion of the event-by-event (EbyE) flow fluctuations measurements in $\mathrm{A}+\mathrm{A}$ collisions. Finally I discuss open issues and future physics opportunities.

\section{Collectivity in small systems}

The observation of collective behaviour in high-multiplicity $p+\mathrm{A}$ collisions [3] came as a surprise, since the transverse size of the produced system was thought to be too small for the hydrodynamic description to work. Experimentalists attempt to elucidate this puzzle by mapping out the detailed properties of the azimuthal harmonics in $p+\mathrm{A}$ collisions and comparing with those in peripheral $\mathrm{A}+\mathrm{A}$ collisions.

Figure 1 (a) shows the first five azimuthal harmonics $v_{1}$ to $v_{5}$ as a function of $p_{\mathrm{T}}$ in high-multiplicity $p+\mathrm{Pb}$ collisions, obtained from a two-particle correlation (2PC) method [4]. The away-side and near-side short-range correlations have been estimated from events with low multiplicity and subtracted. The $v_{n}$ magnitude is largest for $n=2$, and decreases with increasing $n$. All $v_{n}$ increase with $p_{\mathrm{T}}$ up to 3-5 GeV and then decrease, but remain positive at higher $p_{\mathrm{T}}$. It is interesting that the $v_{2}$ and $v_{3}$ values are sizable at $p_{\mathrm{T}}>8 \mathrm{GeV}(3-5 \%)$, reflecting a significant near-side 

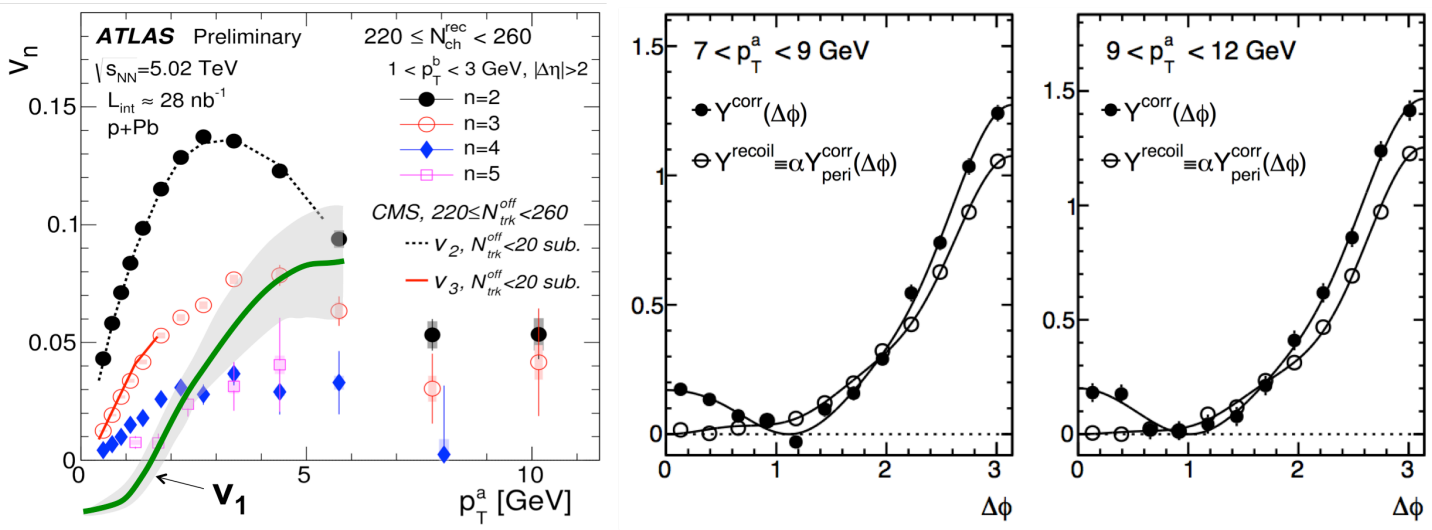

Figure 1. The $v_{n}\left(p_{\mathrm{T}}\right)$ with $n=2$ to 5 for pairs with $|\Delta \eta|>2$ [4]. An overlay sketch of rapidity-even $v_{1}$ data is also shown. Results are compared to the CMS data with comparable multiplicity.

ridge regardless of the recoil subtraction procedure as shown in the right panels of Fig. 1. This may be the first indication of a small azimuthal anisotropy of jet yield in high-multiplicity $p+\mathrm{Pb}$ collisions.

Figure 1 also shows an overlay sketch of the preliminary rapidity-even $v_{1}$ [4]. The values of $v_{1}$ are comparable to $v_{3}$ at $p_{\mathrm{T}} \sim 4 \mathrm{GeV}$, albeit with larger uncertainties since they are directly sensitive to the recoil subtraction.

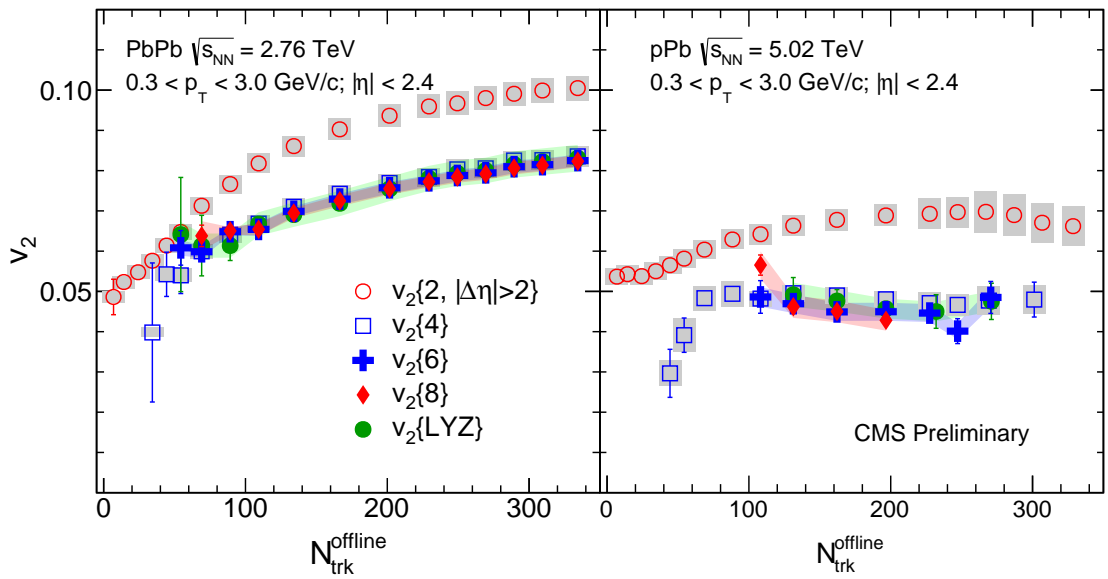

Figure 2. The $v_{2}$ results obtained from two, four, six, eight-particle cumulants, as well as Lee-Yang Zero (LYZ) method, averaged over the $p_{\mathrm{T}}$ range of $0.3-3.0 \mathrm{GeV}$, as a function of number of charged particles in $\mathrm{Pb}+\mathrm{Pb}$ (left panel) and $p+\mathrm{Pb}$ (right panel) collisions [5].

Multi-particle cumulants beyond 2PC have been argued to be able to distinguish the correlation arising from collective dynamics from those arising from sources involving few particles. CMS presents a detailed measurement of multi-particle cumulants $v_{2}\{4\}, v_{2}\{6\}$ and $v_{2}\{8\}$, as well as Lee-Yang Zero (LYZ) method $v_{2}\{\mathrm{LYZ}\}$ in $p+\mathrm{Pb}$ or $\mathrm{Pb}+\mathrm{Pb}$ collisions [5]. The different methods agree within $\pm 10 \%$ over a broad multiplicity range in each collision system (Fig. 2), confirming the collective nature of the observed correlations in both systems. The apparent difference from $v_{2}\{2\}$ reflects the fluctuations either present in the initial geometry or generated in the collective expansion.

Hydrodynamics is the commonly accepted description for $v_{n}$ in central and mid-central A+A collisions. However, as the system size decreases, this description is expected to break down at some point. Comparison of $v_{n}$ between $p+\mathrm{Pb}$ and $\mathrm{Pb}+\mathrm{Pb}$ at similar multiplicity can help us to understand where and how the break down takes place. Figure 3 compares the multiplicity dependence of the integral $v_{3}$ (left panel) and the $p_{\mathrm{T}}$ dependence of $v_{3}$ (right panel) [6] between the two collision systems. Very similar multiplicity and $p_{\mathrm{T}}$ dependence are observed between the two systems, suggesting that the collectivity is controlled by total multiplicity, as expected from a argument based on conformal hydrodynamics [7]. This conformal scaling argument also qualitatively explains the similarity of the multiplicity dependence of the HBT radii in $p+p, p+\mathrm{Pb}$ and $\mathrm{Pb}+\mathrm{Pb}$ collisions [8]. CMS also presents a precision measurement of $v_{2}$ 
and $v_{3}$ for $K_{s}^{0}$ and $\Lambda$ in $p+\mathrm{Pb}$ collisions [9], a clear particle-mass dependence is seen that persists to at least $5 \mathrm{GeV}$ in $p_{\mathrm{T}}$ with a magnitude similar to values in $\mathrm{Pb}+\mathrm{Pb}$ collisions at comparable multiplicity.
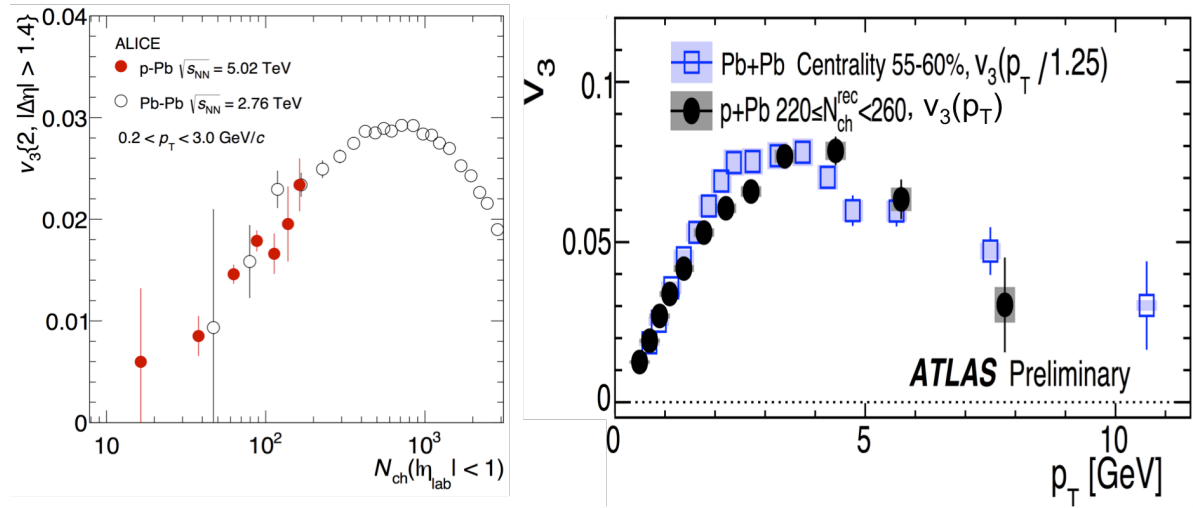

Figure 3. The $v_{3}$ data compared between $p+\mathrm{Pb}$ and $\mathrm{Pb}+\mathrm{Pb}$ collisions as a function of charged particle multiplicity in $|\eta|<1[6]$ (left panel) and as a function of $p_{\mathrm{T}}[4]$ at approximately the same multiplicity (right panel) in the two systems. In the right panel, the $\mathrm{Pb}+\mathrm{Pb}$ data are rescaled horizontally by a constant factor of 1.25 to match the $\left\langle p_{\mathrm{T}}\right\rangle$ in $p+\mathrm{Pb}$ collisions.

Here I would like to caution people about one technical aspect of those 2PC analyses that rely on the ZYAM (zero-yield at minimum) procedure. In this procedure, the near-side excess associated with the long-range ridge is obtained by subtracting a constant pedestal that is matched to the minimum of the correlation function. Due to the large and broad away-side jet peak (especially at low $p_{\mathrm{T}}$ ), the tail of the away-side peak tends to feed into the near-side region and create a minimum at $\Delta \phi_{\text {ZYAM }}=0$. As long as the ridge yield is not enough to fill the near-side jet valley, one always finds zero ridge yield despite the apparent change in the near-side shape of the correlation function (see Fig. 4(a) from [10]). Consequently, the multiplicity dependence of the extracted near-side per-trigger yield exhibits a step-like structure at small $N_{\mathrm{ch}}$ [3] (Fig. 4(b)). This step could be misinterpreted as the onset of ridge or saturation physics.
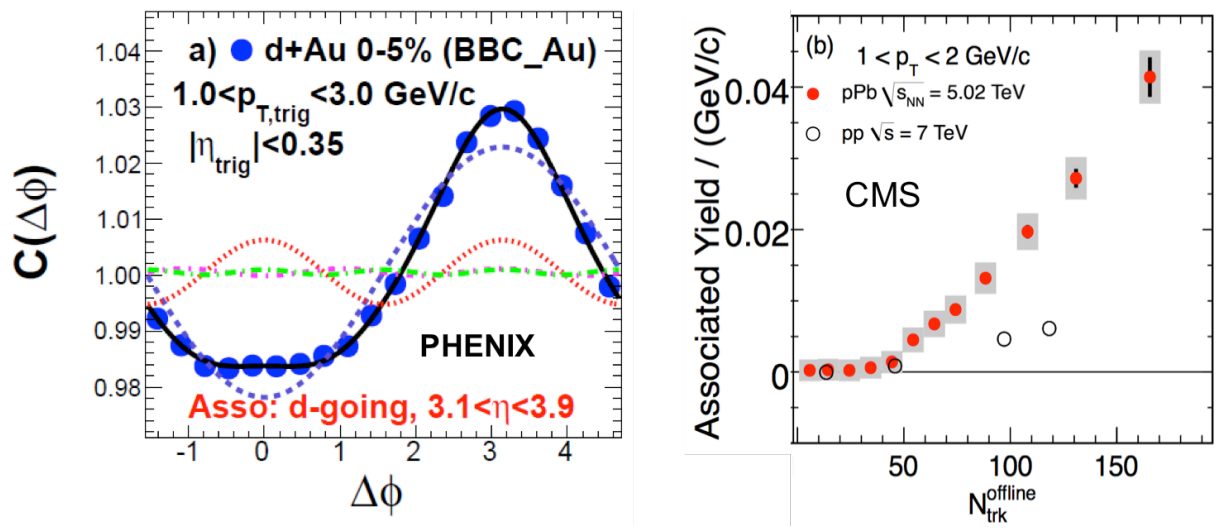

Figure 4. (a) Azimuthal correlations between mid-rapidity hadrons and forward $E_{\mathrm{T}}$ in the d-going direction in $\sqrt{s_{\mathrm{NN}}}=200 \mathrm{GeV} \mathrm{d}+\mathrm{Au}$ collisions [10]. (b) The per-trigger yield for particle pairs selected in $1<p_{\mathrm{T}}<2 \mathrm{GeV}$ as a function of charged particle multiplicity in $\sqrt{s_{\mathrm{NN}}}=2.76$ $\mathrm{TeV} p+\mathrm{Pb}$ collisions [3].

\section{Comments on selected flow results in A+A collisions}

Several new flow results are worthy of mentioning. ALICE publishes a detailed measurement of $v_{2}$ for many identified particles [11]. Comparing to the RHIC results, ALICE results imply stronger radial flow and hadronic rescattering effects. The latter is responsible for a poor number-of-constituent-quark (NCQ) scaling for different particle species. In particular, the NCQ-scaling of $\phi$-meson $v_{2}$ suggests that it flows like a baryon in central collisions and 
like a meson in mid-central collisions. This could happen if $\phi$ meson undergoes significant hadronic re-scattering in central collision where the radial flow is the largest, despite its small hadronic cross-section.

New flow results in $\mathrm{Cu}+\mathrm{Au}$ and $\mathrm{U}+\mathrm{U}$ collisions are reported by PHENIX and STAR [12]. Significant $v_{1}$ is measured in $\mathrm{Cu}+\mathrm{Au}$ collisions with respect to the spectators, reflecting the average dipolar geometry in these collisions. The dependence of the multiplicity dependence of $v_{2}$ in ultra-central $\mathrm{U}+\mathrm{U}$ collisions is found to differ from that in the $\mathrm{Au}+\mathrm{Au}$ collisions, reflecting the influence of the deformed geometry in $\mathrm{U}+\mathrm{U}$ collisions. However, each collision system introduces its own uncertainty in collision geometry, which need to be understood in order to make good use of these new results.

Due to event-by-event fluctuations, it was predicted that the event-plane angle $\Phi_{n}$ fluctuates in $p_{\mathrm{T}}$, leading to a breakdown of the factorization in extracting the $v_{n}$ from azimuthal harmonics $V_{n \Delta}$ in two-particle correlations [13]. This breakdown can be quantified by the $r_{n}$ factor:

$$
r_{n}=\frac{V_{n \Delta}\left(p_{\mathrm{T}}^{\mathrm{a}}, p_{\mathrm{T}}^{\mathrm{b}}\right)}{\sqrt{V_{n \Delta}\left(p_{\mathrm{T}}^{\mathrm{a}}, p_{\mathrm{T}}^{\mathrm{a}}\right) V_{n \Delta}\left(p_{\mathrm{T}}^{\mathrm{b}}, p_{\mathrm{T}}^{\mathrm{b}}\right)}} \approx \frac{\left\langle v_{n}\left(p_{\mathrm{T}}^{\mathrm{a}}\right) v_{n}\left(p_{\mathrm{T}}^{\mathrm{b}}\right) \cos n\left(\Phi_{n}\left(p_{\mathrm{T}}^{\mathrm{a}}\right)-\Phi\left(p_{\mathrm{T}}^{\mathrm{b}}\right)\right)\right\rangle}{\sqrt{\left\langle v_{n}^{2}\left(p_{\mathrm{T}}^{\mathrm{a}}\right)\right\rangle\left\langle v_{n}^{2}\left(p_{\mathrm{T}}^{\mathrm{b}}\right)\right\rangle}}
$$

$r_{n}$ is expected to be less than one if $\Phi_{n}$ depends on $p_{\mathrm{T}}$. Experimental data show significant factorization breaking for $v_{2}$ in ultra-central $\mathrm{Pb}+\mathrm{Pb}$ collisions on the order of up to $20 \%$ ( $r_{2}$ reaches 0.8$)$, consistent with viscous hydrodynamic model predictions [14]. However, the breaking is much smaller for $v_{2}$ in other centrality ranges and for other harmonics in the full centrality range $[14,15]$. New data in $p+\mathrm{Pb}$ collisions from CMS and ALICE suggest the level of breaking is at most a few percents in low $p_{\mathrm{T}}$ region [16]. But even some of these deviations could be due to nonflow effects from jets, hence the use of recoil subtraction procedure [4] could be important.

The $v_{n}$ spectrum in ultra-central $\mathrm{A}+\mathrm{A}$ collisions poses a challenge for theory. The original motivation is that the initial conditions in these collisions are predominantly generated by fluctuations such that the magnitudes of first several $\varepsilon_{n}$ are comparable, and that the hydrodynamic response is expected to be linear $v_{n} \propto \varepsilon_{n}$ for all harmonics. Hence these collisions are expected to provide better constraints on the mechanism of the density fluctuations. The precision data from ATLAS [15] and CMS [14] show several features that are not described by models: 1) $v_{3}$ is comparable or larger than $v_{2}$, which is challenging since naively one expect $\varepsilon_{3} \approx \varepsilon_{2}$, but the $v_{3}$ should suffer larger viscous correction. 2) The $v_{2}\left(p_{\mathrm{T}}\right)$ has very different shape comparing to other harmonics; It peaks at much lower $p_{\mathrm{T}}$, around $1.5 \mathrm{GeV}$ compare to 3-4 GeV for other harmonics. Calculation in Ref. [17] shows that the nucleon-nucleon correlation and effects of bulk viscosity can reduce the $v_{2}$ values relative to $v_{3}$.

\section{Event-by-event flow fluctuations in $\mathbf{A}+\mathbf{A}$ collisions}

In heavy-ion collisions, the number of participating nucleons $N_{\text {part }}$ is finite and their positions fluctuate randomly in the transverse plane, leading to strong EbyE fluctuations of $\varepsilon_{n}$ and participant plane angle $\Phi_{n}^{*}$. Consequently, the matter created in each collision has it own set of flow harmonics, described by a joint probability distribution (pdf):

$$
p\left(v_{n}, v_{m}, \ldots ., \Phi_{n}, \Phi_{m}, \ldots .\right)=\frac{1}{N_{\mathrm{evts}}} \frac{d N_{\mathrm{evts}}}{d v_{n} d v_{m} \ldots d \Phi_{n} d \Phi_{m} \ldots} .
$$

Some projections of this pdf, such as $p\left(v_{n}\right)$, can be measured directly via unfolding method [18]. But they are more generally studied via $m$-particle correlations of the following form [19]:

$$
\left\langle\cos \left(n_{1} \phi_{1}+n_{2} \phi_{2} \ldots+n_{m} \phi_{m}\right)\right\rangle=\left\langle v_{n_{1}} v_{n_{2}} \ldots v_{n_{m}} \cos \left(n_{1} \Phi_{n_{1}}+n_{2} \Phi_{n_{2}} \ldots+n_{m} \Phi_{n_{m}}\right)\right\rangle, \Sigma n_{i}=0 .
$$

For example (more can be found in Ref. [21]), the two-particle cumulant of $p\left(v_{n}\right)$ is obtained as $\left\langle\cos \left(n \phi_{1}-n \phi_{2}\right)\right\rangle=$ $\left\langle v_{n}^{2} \cos \left(n \Phi_{n}-n \Phi_{n}\right)\right\rangle=\left\langle v_{n}^{2}\right\rangle$; the lowest-order cumulant for $p\left(v_{n}, v_{m}\right)$ requires a four-particle correlation of the form [20]:

$\left\langle\cos \left(n \phi_{1}-n \phi_{2}+m \phi_{3}-m \phi_{4}\right)\right\rangle_{c}=\left\langle\cos \left(n \phi_{1}-n \phi_{2}+m \phi_{3}-m \phi_{4}\right)\right\rangle-\left\langle\cos \left(n \phi_{1}-n \phi_{2}\right)\right\rangle\left\langle\cos \left(m \phi_{3}-m \phi_{4}\right)\right\rangle=\left\langle v_{n}^{2} v_{m}^{2}\right\rangle-\left\langle v_{n}^{2}\right\rangle\left\langle v_{m}^{2}\right\rangle ;$

the event-plane correlations $p\left(\Phi_{n}, \Phi_{m}, \ldots\right)$ can be accessed via a $\Sigma c_{i}$-particle correlation of the form:

$$
\left\langle\cos \left(\Sigma_{i_{1}=1}^{c_{1}} \phi_{i_{1}}+\Sigma_{i_{2}=1}^{c_{2}} 2 \phi_{i_{2}}+\ldots+\Sigma_{i_{l}=1}^{c_{l}} l \phi_{i_{l}}\right)\right\rangle=\left\langle v_{1}^{c_{1}} v_{2}^{c_{2}} . . v_{l}^{c_{l}} \cos \left(c_{1} \Phi_{1}+2 c_{2} \Phi_{2} \ldots+l c_{l} \Phi_{l}\right)\right\rangle, \quad \Sigma i c_{i}=0 .
$$


In the last few years, impressive progresses have been made in studying these flow observables [21]. We now understand that $v_{2}$ and $v_{3}$ are the dominant harmonics, driven mainly by the linear response to the ellipticity and triangularity of the initially produced fireball [22]: $v_{2} e^{i 2 \Phi_{2}} \propto \varepsilon_{2} e^{i 2 \Phi_{2}^{*}}, v_{3} e^{i 3 \Phi_{3}} \propto \varepsilon_{3} e^{i 3 \Phi_{3}^{*}}$. In contrast, the higher-order harmonics $v_{4}, v_{5}$ and $v_{6}$ arise from both the initial geometry and non-linear mixing of lower-order harmonics [23, 24]:

$$
\begin{aligned}
& v_{4} e^{i 4 \Phi_{4}}=a_{0} \varepsilon_{4} e^{i 4 \Phi_{4}^{*}}+a_{1}\left(\varepsilon_{2} e^{i 2 \Phi_{2}^{*}}\right)^{2}+\ldots=c_{0} e^{i 4 \Phi_{4}^{*}}+c_{1} v_{2}^{2} e^{i 4 \Phi_{2}}+\ldots, \\
& v_{5} e^{i 5 \Phi_{5}}=a_{0} \varepsilon_{5} e^{i 5 \Phi_{5}^{*}}+a_{1} \varepsilon_{2} e^{i 2 \Phi_{2}^{*}} \varepsilon_{3} e^{i 3 \Phi_{3}^{*}}+\ldots=c_{0} e^{i 5 \Phi_{5}^{*}}+c_{1} v_{2} v_{3} e^{i\left(2 \Phi_{2}+3 \Phi_{3}\right)}+\ldots \\
& v_{6} e^{i 6 \Phi_{6}}=a_{0} \varepsilon_{6} e^{i 6 \Phi_{6}^{*}}+a_{1}\left(\varepsilon_{2} e^{i 2 \Phi_{2}^{*}}\right)^{3}+a_{2}\left(\varepsilon_{3} e^{i 3 \Phi_{3}^{*}}\right)^{2}+a_{3} \varepsilon_{2} e^{i 2 \Phi_{2}^{*}} \varepsilon_{4} e^{i 4 \Phi_{4}^{*}}+\ldots=c_{0} e^{i 6 \Phi_{6}^{*}}+c_{1} v_{2}^{3} e^{i 6 \Phi_{2}}+c_{2} v_{3}^{2} e^{i 6 \Phi_{3}}+\ldots
\end{aligned}
$$

Furthermore, the measured $p\left(v_{2}\right)$ distributions show significant deviation in the tail from Bessel-Gaussian function[18], suggesting either the $p\left(\varepsilon_{2}\right)$ is non-Gaussian [25] or the response of $v_{2}$ to $\varepsilon_{2}$ is not strictly linear [26]. New ATLAS results show that the non-Gaussian behavior is captured by $<1-2 \%$ difference between $v_{2}\{4\}$ and $v_{2}\{6\}$, suggesting that $v_{n}\{2 k\}$ for $k>1$ are not very sensitive to flow fluctuations given their current systematic uncertainties [27, 21].

The EbyE flow fluctuations can also be studied using various event-shape selection methods [28, 29]. The performances of these methods have been validated in a AMPT model framework $[29,30]$ and applied in the ATLAS data analysis [31]. In this analysis, the azimuthal angle distribution of the transverse energy $E_{\mathrm{T}}$ in the forward calorimeter over $3.3<|\eta|<4.9$ is expanded into a Fourier series for each event:

$$
2 \pi \frac{d E_{\mathrm{T}}}{d \phi}=\Sigma E_{\mathrm{T}}\left(1+2 \Sigma_{n=1}^{\infty} q_{n} \cos n\left(\phi-\Psi_{n}\right)\right)
$$

where the reduced flow vector $q_{n}$ represents the $E_{\mathrm{T}}$-weighted raw flow coefficients $v_{n}^{\mathrm{obs}}, q_{n}=\Sigma\left(E_{\mathrm{T}} v_{n}^{\mathrm{obs}}\right) / \Sigma E_{\mathrm{T}}$. Events are first divided according to $\Sigma E_{\mathrm{T}}$ into centrality classes, events in each class are further divided according to the $q_{2}$. This classification separates events with similar multiplicity but with very different ellipticity. The values of $v_{n}$ are then calculated at mid-rapidity $(|\eta|<2.5)$ using a $2 \mathrm{PC}$ method.

The correlation of $v_{2}$ between two different $p_{\mathrm{T}}$ ranges from ATLAS, presented in Fig. 5(a), shows a boomeranglike centrality dependence due to a stronger viscous correction for $v_{2}$ at higher $p_{\mathrm{T}}$. In contrast, the correlation within a narrow centrality interval is found to be always linear, suggesting that viscous effects are controlled by the system size not its overall shape. The $v_{3}-v_{2}$ correlations, shown in Fig. 5(b), reveal an anti-correlation that is similar in magnitude to the correlation between $\varepsilon_{3}$ and $\varepsilon_{2}$ (see Fig. 6(a)), implying that this correlation reflects mostly initial geometry effects. ATLAS also studied $v_{4}-v_{2}$ (Fig. 5(c)) and $v_{5}-v_{2}$ correlations. The patterns in these correlations are found to be well described by two-parameter fits of the following form, motivated by interplay between the linear and non-linear
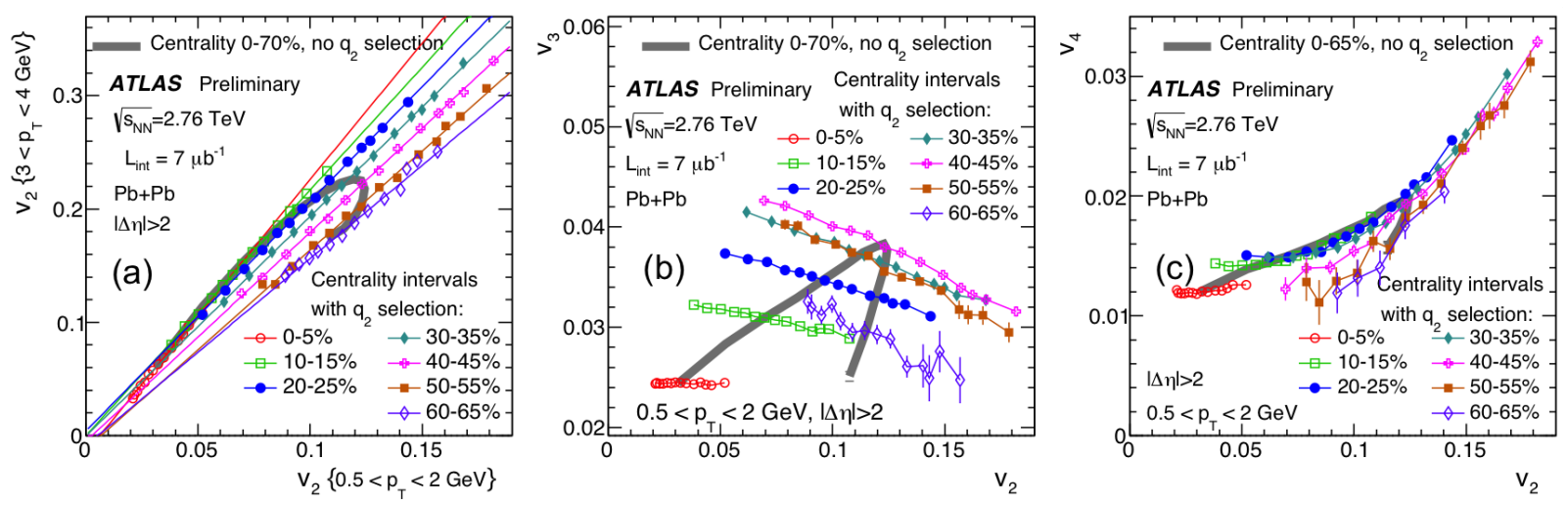

Figure 5. The correlation of (panel a) $v_{2}$ in two different $p_{\mathrm{T}}$ ranges, (panel b) $v_{3}$ and $v_{2}$ in the same $p_{\mathrm{T}}$ range and (panel c) $v_{4}$ and $v_{2}$ in the same $p_{\mathrm{T}}$ range. The data points in each centrality interval correspond to 14 different ellipticity classes selected via an event-shape engineering technique. These data are overlaid with the centrality dependence without event-shape selection (think grey lines). The thin solid straight lines in the left panel represent a linear fit of the data in each centrality. Results taken from Ref. [31]. 

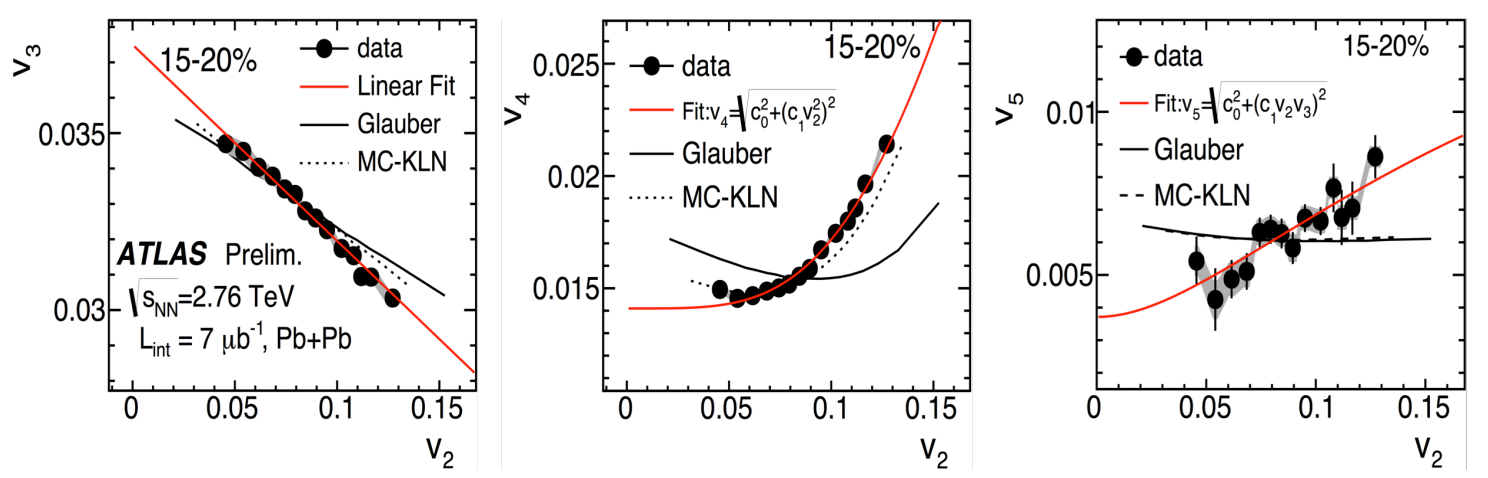

Figure 6. The $v_{3}-v_{2}$ (left), $v_{4}-v_{2}$ (middle) and $v_{5}-v_{2}$ (right) correlations measured in $0.5<p_{\mathrm{T}}<2 \mathrm{GeV} 10-15 \%$ centrality interval. The correlation data are fit to functions that include both linear and non-linear contributions. The correlation data are also compared with re-scaled $\varepsilon_{n}-\varepsilon_{2}$ correlation from the MC Glauber and MC-KLN models. Results taken from Ref. [31].
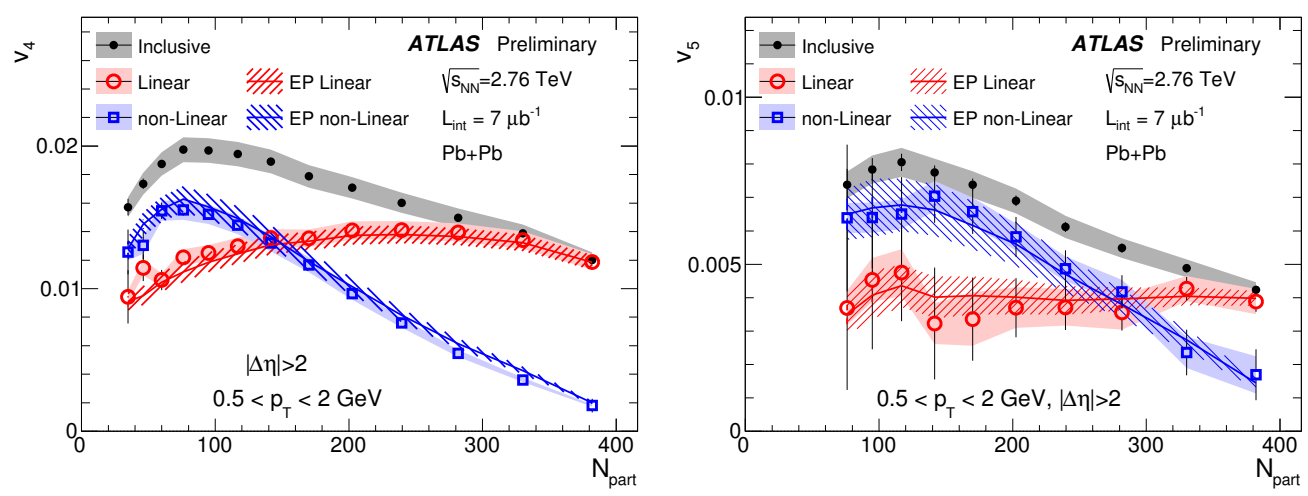

Figure 7. The centrality dependence of the $v_{4}$ (left) and $v_{5}$ (right) in $0.5-2 \mathrm{GeV}$ and the associated linear and non-linear components extracted from the fit in Fig. 6. They are compared with the linear and non-linear components estimated from the previous published event-plane correlations [24]. Results taken from Ref. [31].

collective dynamics given by Eq. 6 :

$$
v_{4}=\sqrt{c_{0}^{2}+\left(c_{1} v_{2}^{2}\right)^{2}}, v_{5}=\sqrt{c_{0}^{2}+\left(c_{1} v_{2} v_{3}\right)^{2}},
$$

These fits allow ATLAS to decompose the $v_{4}$ and $v_{5}$, centrality by centrality, into linear and non-linear terms as $v_{n}^{\mathrm{L}}=c_{0}$ and $v_{n}^{\mathrm{NL}}=\sqrt{v_{n}^{2}-c_{0}^{2}}$, respectively, as shown in Fig. 7. The linear term associated with $\varepsilon_{n}$ depends only weakly on centrality, and dominates the $v_{n}$ signal in central collisions. The excellent agreement with a similar decomposition based on the measured EP correlations [24] implies that the correlations between flow magnitudes arise mostly from the correlations between the flow angles.

\section{Future prospects}

The application of event-shape selection techinique is not only limited to flow observables. It should be straightforward to apply this technique to other observables, such as HBT correlation, $R_{\mathrm{AA}}$, dihadron correlation, chiral magnetic effects and more. A study by PHENIX [32] shows that the measured $2^{\text {nd }}$-order freezeout eccentricity $\varepsilon_{2}^{\mathrm{f}}$ is strongly correlated with selection on the ellipticity. This is qualitatively expected, as events with large $v_{2}$ should on average have large $\varepsilon_{2}$, and hence they may have larger $\varepsilon_{2}^{\mathrm{f}}$.

One open issue is the response or back-reaction of the medium to the energy deposited by jets. CMS results suggest that the lost energy of very high $p_{\mathrm{T}}$ jets is transported to very large angle and to low $p_{\mathrm{T}}$ particles [33], but not much is know about the back-reaction of the mini-jets that have the energy of a few $\mathrm{GeV}$ to few tens of $\mathrm{GeV}$. These mini-jets are abundantly produced and dominate particle spectrum at intermediate $p_{\mathrm{T}}$. The back-reaction 

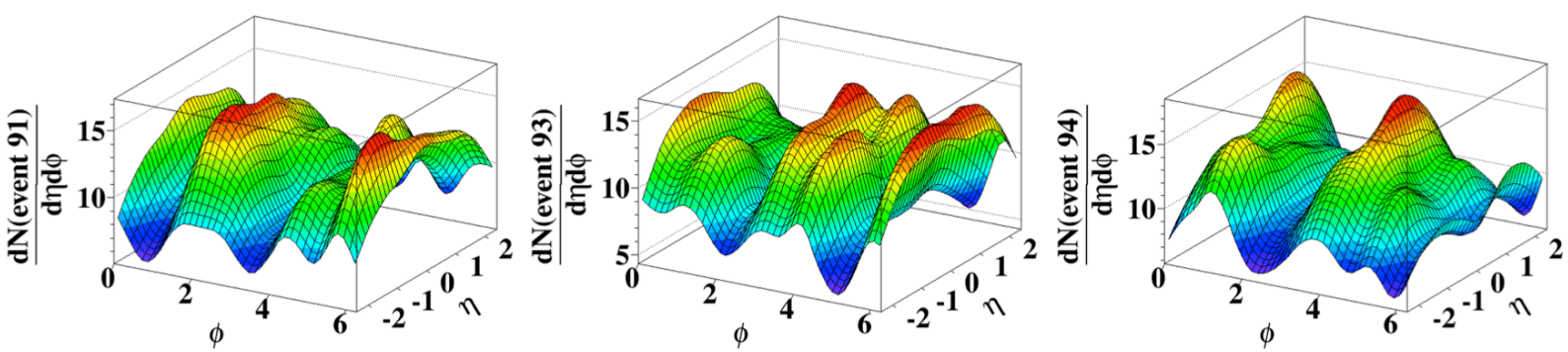

Figure 8. The distributions of the particles in $\eta \times \phi$ space for three typical Au+Au collisions at $\sqrt{s_{\mathrm{NN}}}=200 \mathrm{GeV}$ in $0-10 \%$ centrality interval, obtained from 3+1D calculation based on ideal hydrodynamics [34].

of mini-jets was historically studied using 2PC analysis aided by flow background subtraction, which suffers large systematic uncertainties due to dominance of collective flow in the correlation structure. We can improve the situation by performing the analysis in events selected to have small $v_{n}$. But ultimately, 2PC method may not be the best approach to investigate the jet-medium interactions. Instead, we need methods that are sensitive to the localized $\eta \times \phi$ structures in the EbyE particle multiplicity or $E_{\mathrm{T}}$ distributions. To motivate this idea, Fig. 8 shows the EbyE $\eta \times \phi$ distribution of particle density from a 3+1D hydrodynamic calculation based on the AMPT initial condition [34]. The localized peaks and valleys in these events could be remnant of the mini-jets in the initial state. These localized structures, or "hydro-jets", are much broader than typical high- $p_{\mathrm{T}}$ jets. They can be found, possibly as fake-jets, by running standard jet reconstruction algorithm. Obviously, the jet finding algorithm may need to be modified in order to maximize the finding efficiency and better adapt to the shape of these objects. One can then perform a detailed study of the spectrum and substructure of these hydro-jets.

Most studies in $\mathrm{A}+\mathrm{A}$ collisions consider only fluctuations in the transverse plane, and dynamics in the longitudinal direction is often assumed to be boost invariant. However, a Glauber model calculation [30] shows that the number of participating nucleons and eccentricity vectors $\vec{\varepsilon}_{n} \equiv\left(\varepsilon_{n}, \Phi_{n} *\right)$, defined separately for the two colliding nuclei, can differ strongly on EbyE basis due to fluctuations: $N_{\text {part }}^{\mathrm{F}} \neq N_{\text {part }}^{\mathrm{B}}$ and $\vec{\varepsilon}_{n}^{\mathrm{F}} \neq \vec{\varepsilon}_{n}^{\mathrm{B}}$. Furthermore, the energy deposition for each participating nucleon is not symmetric: Particles in the forward (backward) rapidity are preferably produced by the participants in the forward-going (backward-going) nucleus [35]. Due to these two effects, the eccentricity vector and the flow vector $\vec{v}_{n} \equiv\left(v_{n}, \Phi_{n}\right)$ of the initially produced fireball should depend on $\eta$ (see Fig. 9) [30]:

$$
\vec{v}_{n}(\eta) \propto \vec{\varepsilon}_{n}^{\mathrm{tot}}(\eta) \approx \alpha(\eta) \vec{\varepsilon}_{n}^{\mathrm{F}}+(1-\alpha(\eta)) \vec{\varepsilon}_{n}^{\mathrm{B}} \equiv \varepsilon_{n}^{\mathrm{tot}}(\eta) e^{i n \Phi_{n}^{* \mathrm{tot}}(\eta)}, \alpha(\eta) \approx \frac{f(\eta)}{f(\eta)+f(-\eta)}, n=2 \text { or } 3
$$

where $\alpha(\eta)$ is an $\eta$-dependent weight factor for the forward-going participating nucleons, and $f(\eta)$ is the average emission profile per-nucleon [35]. This relation has been verified using the Glauber model and AMPT model. The asymmetry between $\vec{\varepsilon}_{n}^{\mathrm{F}}$ and $\vec{\varepsilon}_{n}^{\mathrm{B}}$ is very large and it is shown to convert into a similar asymmetry between the $\vec{v}_{n}$ in
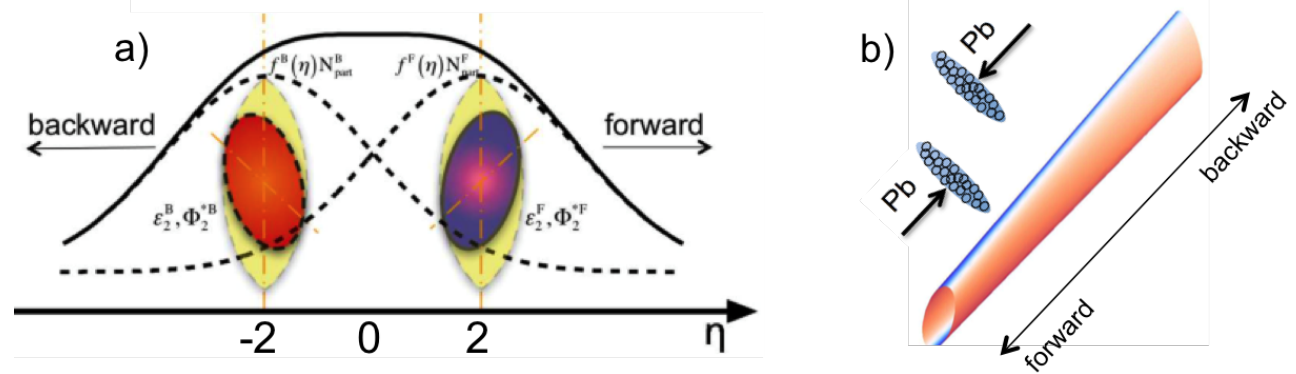

Figure 9. Schematic illustration of the forward-backward fluctuation of second-order eccentricity and participant plane in A+A collisions. The dashed-lines indicate the particle production profiles for forward-going and backward-going participants, respectively [30, 36]. The right panel shows the longitudinal view of the twisted fireball. 
forward and backward $\eta$. This $\mathrm{F} / \mathrm{B}$ asymmetry is expected to be particularly large for $p+\mathrm{Pb}$ collisions, and initial indication of such $\mathrm{F} / \mathrm{B}$ asymmetry has indeed been observed in $p+\mathrm{Pb}$ collisions [37].

\section{Summary}

The Quark Matter 2014 conference has seen impressive progresses in the study of collective behavior in high energy nuclear collisions. Significant azimuthal harmonics have been measured for $v_{1}$ to $v_{5}$ in $p+$ A collisions over broad range of $p_{\mathrm{T}}$ and event multiplicity. The $v_{2}$ signal from multi-particle correlations is large, suggesting that the ridge in $p+\mathrm{A}$ collisions reflects the genuine collectivity of the produced medium. Remarkable agreement of the $v_{n}\left(p_{\mathrm{T}}, N_{\mathrm{ch}}\right)$ between $p+\mathrm{A}$ and $\mathrm{A}+\mathrm{A}$ collisions suggests that the observed collectivity is driven by similar dynamics in the two systems. Several new flow results in A+A collisions, such as $v_{n}$ for different particle species and collision systems and $v_{n}$ in ultra-central collisions, serve to refine the hydrodynamic models. Important progresses have also been made in measuring a large class of event-by-event flow observables, in particular a brand-new detailed study of the $v_{2}-v_{2}$ and $v_{n}-v_{2}$ correlations using the event-shape selection technique. A coherent picture of initial condition and collective flow based on linear and non-linear hydrodynamic responses is derived, which qualitatively describes most results on these observables. Several new types of fluctuation measurements should be pursued, which can further our understanding of the event-shape fluctuations and collective expansion dynamics.

This research is supported by NSF under grant number PHY-1305037 and by DOE through BNL under grant number DE-AC02-98CH10886.

\section{References}

[1] U. Heinz and R. Snellings, Ann. Rev. Nucl. Part. Sci. 63, 123 (2013).

[2] M. Luzum and H. Petersen, J. Phys. G 41, 063102 (2014).

[3] CMS Collaboration, Phys. Lett. B 718, 795 (2013), Phys. Lett. B 724, 213 (2013); ALICE Collaboration, Phys. Lett. B 719, 29 (2013),Phys. Lett. B 726, 164 (2013); ATLAS Collaboration, Phys. Rev. Lett. 110, 182302 (2013), Phys. Lett. B 725, 60 (2013); PHENIX Collaboration, Phys. Rev. Lett. 111, 212301 (2013).

[4] S. Radhakrishnan, these proceedings.

[5] Q. Wang, these proceedings.

[6] A. Timmins, these proceedings.

[7] G. Basar and D. Teaney, arXiv:1312.6770 [nucl-th].

[8] D. Gangadharan, these proceedings; Dogra, these proceedings.

[9] M. Sharma, these proceedings.

[10] S. Huang, these proceedings.

[11] ALICE Collaboration, arXiv:1405.4632 [nucl-ex].

[12] H. Nakagomi, these proceedings; H. Wang, these proceedings.

[13] F. G. Gardim, F. Grassi, M. Luzum and J. -Y. Ollitrault, Phys. Rev. C 87, no. 3, 031901 (2013)

[14] CMS Collaboration, JHEP 1402, 088 (2014).

[15] ATLAS Collaboration, Phys. Rev. C 86, 014907 (2012).

[16] D. Devetak, these proceedings; Y. Zhou, these proceedings.

[17] G. Denicol, these proceedings.

[18] ATLAS Collaboration, JHEP 1311, 183 (2013); J. Jia and S. Mohapatra, Phys. Rev. C 88, 014907 (2013).

[19] R. S. Bhalerao, M. Luzum and J. -Y. Ollitrault, Phys. Rev. C 84, 034910 (2011)

[20] A. Bilandzic, C. H. Christensen, K. Gulbrandsen, A. Hansen and Y. Zhou, Phys. Rev. C 89, 064904 (2014)

[21] J. Jia, arXiv:1407.6057 [nucl-ex].

[22] Z. Qiu and U. W. Heinz, Phys. Rev. C 84, 024911 (2011);

[23] F. G. Gardim, et al., Phys. Rev. C 85, 024908 (2012);D. Teaney and L. Yan, Phys. Rev. C 86, 044908 (2012).

[24] ATLAS Collaboration, arXiv:1403.0489 [hep-ex].

[25] L. Yan, these proceedings.

[26] H. Niemi, these proceedings.

[27] D. Derendarz, these proceedings.

[28] J. Schukraft, A. Timmins and S. A. Voloshin, Phys. Lett. B 719, 394 (2013).

[29] P. Huo, J. Jia and S. Mohapatra, arXiv:1311.7091 [nucl-ex].

[30] J. Jia and P. Huo, arXiv:1402.6680 [nucl-th], arXiv:1403.6077 [nucl-th].

[31] S. Mohapatra, these proceedings, ATLAS-CONF-2014-022, http://cdsweb.cern.ch/record/1702980

[32] S. Esumi, these proceedings.

[33] CMS Collaboration, Phys. Rev. C 84, 024906 (2011)

[34] X.N. Wang and L. Pang, poster and private communications.

[35] A. Bialas and K. Zalewski, Phys. Rev. C 82, 034911 (2010) [Erratum-ibid. C 85, 029903 (2012)] [arXiv:1008.4690 [hep-ph]].

[36] P. Bozek, W. Broniowski and J. Moreira, Phys. Rev. C 83, 034911 (2011).

[37] L. Xu, these proceedings. 
Ayailable online at www.sciencedirect.com sdilog-3p.pdit

$\because$ ScienceDirect 


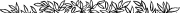
Fif

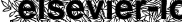
H.

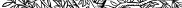
if

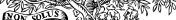

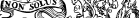

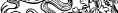
(4) g8)

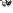
$\frac{1}{4} \frac{2}{4}$ 2.t.

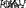
i.

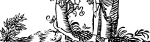
recis

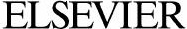

